ISSN: 1130-3743 - eISSN: 2386-5660

DOI: http://dx.doi.org/10.14201/teoredu302155175

\title{
PRÁCTICAS DE LECTURA EN LOS CENTROS Y PROGRAMAS DE ESCRITURA ACADÉMICA
}

\author{
Reading practices in academic writing centers and programs
}

\section{Des pratiques de lecture dans les centres et programmes d'écriture académique}

\author{
Lina CALLE-ARANGO \\ Pontificia Universidad Católica de Chile. Facultad de Educación. Av. Vicuña \\ Mackenna 4860, Macul. Santiago de Chile.lcalle@uc.cl
}

Fecha de recepción: enero de 2018

Fecha de aceptación: marzo de 2018

\section{RESUMEN}

La historia y evolución de los centros y programas de escritura en la educación superior ha sido un tema ampliamente tratado, y gran cantidad de la literatura al respecto deja en evidencia sus prácticas más comunes. No obstante, poca mención se ha hecho a la comprensión lectora como parte integral de los asuntos que atañen a que estos espacios, aunque de unos años hacia acá ha comenzado a haber un asomo de interés. De cara a ello, en este texto se propone reflexionar sobre la importancia de que estos espacios incluyan en sus prácticas y servicios el desarrollo de la competencia lectora, concibiendo esta como indispensable en la construcción de una adecuada competencia comunicativa y apoyo a la alfabetización académica. Adicionalmente, dejar en evidencia que, aprovechando las características de estos lugares, desde la puesta en práctica de una comprensión lectora se promueva la autonomía dentro de la formación académica en géneros discursivos.

Palabras clave: comprensión de lectura; educación superior; alfabetización académica; escritura académica; aprendizaje autodirigido. 


\section{SUMMARY}

The history and evolution of writing centers and programs in higher education has been a widely discussed topic, and much of the literature in this respect reveals its most common practices. However, little mention has been made of reading comprehension as an integral part of the issues that concern these spaces, although a few years ago there has begun to be a hint of interest. In order to do so, this text intends to reflect on the importance of these places to include in their practices and services the development of reading competence, conceiving this as indispensable in the construction of an adequate communicative competence and support for academic literacy. In addition, letting in evidence that, taking advantage of the characteristics of these places, from the implementation of a reading comprehension will promote autonomy within the academic formation in discursive genres.

Key words: reading comprehension; higher education; academic literacy; academic writing; self-directed learning.

\section{SOMMAIRE}

L'histoire et l'évolution des centres et programmes d'écriture dans l'enseignement supérieur ont été un sujet largement discuté et une grande partie de la littérature à cet égard révèle ses pratiques les plus courantes. Cependant, il a été fait peu de mention de la compréhension de la lecture comme partie intégrante des problèmes qui concernent ces espaces, bien qu'il y a quelques années, cela a commencé à susciter un intérêt. Pour ce faire, ce texte a l'intention de réfléchir à l'importance des centres d'écriture pour inclure dans leurs pratiques et services le développement de la compétence en lecture, en concevant cela comme indispensable dans la construction d'une compétence communicative adéquate et soutien à l'alphabétisation scolaire. De plus, en montrant que, en profitant des caractéristiques de ces lieux, l'implémentation d'une compréhension de la lecture favorisera l'autonomie au sein de la formation académique dans les genres discursifs.

Mots clés: compréhension de la lecture; enseignement supérieur; l'alphabétisation scolaire; écriture académique; apprentissage autodirigé.

\section{INTRODUCCIÓN}

Los orígenes y la trayectoria de los centros y programas de escritura en la educación superior es un tema ampliamente tratado; rastrear esta historia desde Norteamérica no resulta difícil; ni desde el nacimiento de estos espacios durante las primeras décadas del siglo xx (Molina, 2014), en principio bajo la denominación de laboratorios o clínicas, y en los cuales se buscaba solventar las dificultades lingüísticas de los estudiantes (Moore, 1995), ni en su momento de mayor proliferación en este país, durante la segunda década del siglo Xx, tras las políticas de admisión 
abierta (Boquet, 1999; Carino, 1996). Información más reciente -finales del siglo xx y comienzos del XxI- se puede encontrar también sobre la inclusión de este modelo a lo largo de Europa, Asia (Tzu-Shan, 2017; Johnston, Cornwell y Yoshida, 2008) y Latinoamérica (Molina, 2014, 2015; Moyano, 2004; Carlino, 2002), algunos con ciertas diferencias con respecto al modelo norteamericano.

A su vez, una gran parte de esta literatura deja en evidencia, desde variadas perspectivas, cuáles han sido sus prácticas más comunes. Resalta el uso de la figura de tutorías, mediante la cual se materializa la noción de personalización, idea primordial alrededor de la cual se construye la idea del centro de escritura (Calle Arango, Pico y Murillo, 2017). Lo anterior con el objetivo, desde sus inicios, de romper con las estructuras jerárquicas del ejercicio tradicional de las aulas de clase (Bruffee, 2008), y que, para muchos estudiantes, representan una presión en su aprendizaje, o bien una estrategia inadecuada para sus procesos cognitivos.

No obstante, brilla por su poca presencia la mención sobre el desarrollo de la comprensión lectora como parte integral de los asuntos que atañen a estos espacios, o al menos en la promoción de estos espacios aún no se constituye como un elemento a ser destacado de manera expresa, como se verá más adelante. Ello pese a que, si se pretende formar un estudiante crítico y competente desde lo comunicativo $-\mathrm{y}$, por tanto, prestar un apoyo transversal desde este lugar- la lectura resulta un elemento indispensable dentro de esta formación, en tanto que involucra procesos cognitivos para la construcción de una visión crítica del mundo y de la información (Flotts, Manzi, Polloni, Carrasco y Zambra, 2016; Griswold, 2006; Ballester y Ibarra, 2015).

De cara a lo anterior, el presente texto tiene el propósito enfatizar en la importancia de que los centros y programas de escritura presten mayor atención, dentro de sus prácticas y servicios, al desarrollo de la competencia lectora crítica, concibiendo esta como parte integral del proceso de construcción de preparación académica. Así mismo, dejar en evidencia que, aprovechando las características de estos espacios, que facilitan el seguimiento de un proceso personalizado, en la puesta en práctica de una comprensión lectora se debe promover la autonomía como pilar de la formación académica e intelectual.

\section{2. ¿¿DÓNDE ESTÁ LA LECTURA EN LOS CENTROS Y PROGRAMAS DE ESCRITURA?}

Griswold, en un artículo mediante el cual buscaba determinar las actitudes y el conocimiento de un "típico» personal del centro de escritura, puso en evidencia lo poco que se ha escrito sobre la aplicación de la teoría de la lectura a la enseñanza y el aprendizaje al interior de los centros de escritura, pese a que muchas de las tareas de escritura de la universidad requieren de copiosa lectura crítica (Griswold, 2006). Aunque diez años después el escenario no resulta ser muy distinto, la verdad es que, pese a que poco se hable en la literatura de la atención de esta habilidad desde estos espacios - pues en otros, tales como los cursos propedéuticos y otras asignaturas para estudiantes de educación superior, es posible percibir una mayor 
inclinación por esta habilidad-, en la práctica parece ya haber un asomo de este interés, aunque aún no sea significativo, o bien no se encuentra lo suficientemente difundido.

El hecho de que autores como Johnston, Cornwell y Yoshida señalen que los centros y programas de escritura asiáticos mantengan como diferencia con la tradición norteamericana que los primeros prestan mayor atención a otras habilidades además de la escrita (Johnston, Cornwell y Yoshida, 2008) deja en entrever una consciencia sobre la importancia de tener en cuenta otras competencias en pro a desarrollar una competencia comunicativa integral. Así mismo, una comparación realizada por Tan en el 2011 entre los centros y programas de escritura norteamericanos y los que él llama no norteamericanos -que, en su texto, refieren a europeos y asiáticos- señala como una similitud entre estos que, en su mayoría, el foco principal recae sobre la escritura académica, pero destaca que algunos de ellos también incluyen expresión oral, así como lectura y escritura para fines específicos (Tan, 2011). Lo anterior coincide con la afirmación que hace Muriel Harris a la hora de caracterizar los centros de escritura norteamericanos: "While most writing centers work only with writing skills, some also offer help with reading, study, and/or oral communication skills" (Harris, 1988) [énfasis propio], entre ellas Purdue University.

Otra evidencia de la existencia de esta atención -pero también de que aún es insuficiente- en Oriente es la tabla que Johnston, Cornwell y Yoshida presentan como resultado de un panel de coordinadores de centros de escritura en Asia y Japón en el marco del JALT2009 Conference Proceedings en 2009. En ella, de los seis centros presentados, dos -Osaka Jogakuin College y Hokusei Gakuen University Junior College, Hokkaido- ya señalan que sus servicios se expanden a otras habilidades distintas, como la lectora (Johnston, Cornwell y Yoshida, 2010, 696). En contraste, sin embargo, la tabla que presentó recientemente Tzu-Shan ofreciendo la información de los centros y programas de escritura de las universidades públicas tradicionales en Taiwán (Tzu-Shan, 2017) evidencia, en la columna de servicios ofrecidos, que ninguno de estos menciona apoyo en las habilidades de comprensión lectora.

En el marco de la educación superior en España, puntualmente, el Centro de Escritura de la Universidad de Cádiz, pese a que su misión enfatiza en la expresión escrita, destaca en su página web que sus miembros colaboran con el Plan Universitario en Fomento del libro, la lectura y la Escritura. Sin embargo, centros como el de la Escuela de Escritura de la Universidad de Alcalá; Centro de Redacción de la Universidad Pompeu Fabra; Centro de Escritura de la Facultad de Filosofía y Letras de la Universidad de Navarra; o el Centro de Escritura de la Universidad Autónoma de Madrid no dejan ver en sus páginas de consulta una atención expresa a esta habilidad, dado que, en términos generales, su énfasis recae en la escritura. Todo lo anterior, como se mencionó arriba, respecto específicamente de los programas y centros de escritura, lo que no excluye la existencia de prácticas de comprensión lectora en asignaturas propias del currículo y vinculadas al desarrollo 
de competencias personales, aunque en muchas ocasiones, nuevamente, con una visión de la lectura supeditada a la producción escrita.

Núñez (2013), coordinador de uno de estos espacios en el marco español, estudió las similitudes y diferencias entre algunos centros (y programas, aunque no lo haga expreso) de Iberoamérica, artículo en el que incluyó algunos centros de Colombia, Argentina, España, México y Perú. Aunque nuevamente los centros y programas presentados poco evidencian dinámicas que hagan especial énfasis en la comprensión lectora, cabría destacar que el objetivo del Programa de desarrollo de habilidades de lectura y escritura académica a lo largo de la carrera (PRODEAC), de la Universidad Nacional General Sarmiento (UNGS), incluye «familiarizar a los estudiantes con las prácticas de lectura y escritura de géneros académicos propios de las disciplinas que cursan", y en sus objetivos de investigación tiene en cuenta la producción de "materiales didácticos que permitan avanzar en las propuestas de enseñanza de la lectura y la escritura académica y profesional» (Núñez, 2013, 74). Así mismo, el Centro de Escritura de la Universidad de los Andes de Colombia, entre los recursos presentados en su página web, ofrece algunos relacionados con cómo hacer guías de lectura (Núñez, 2013); y el Centro de Escritura Académica y Pensamiento Crítico de la Universidad de las Américas Puebla cuenta con una sección sobre lectura, a través de la cual «los usuarios pueden acceder a información sobre revistas especializadas, clubes de lectura, asociaciones y fundaciones que atienden la lectura» (Núñez, 2013, 88). Por su parte, el Centro de Escritura de la Universidad Javeriana de Cali ofrece asistencia a los profesores en la puesta en marcha de estrategias de lectura en sus asignaturas (Núñez, 2013). Es decir, que estos dejan entrever cierta atención a la importancia de la lectura, a pesar de que aún parecen carecer de dinámicas expresas para prestar apoyo personalizado en el desarrollo de tal habilidad.

Para complementar el panorama en esta región, en el libro Panorama de los centros y programas de escritura en Latinoamérica (Molina, 2015), que presenta información y forma de operación de los centros y programas de escritura en el mundo hispanohablante, también es posible encontrar menciones que dan cuenta de cierta atención a la habilidad de comprensión lectora. Ejemplo de ello, para comenzar, es que tanto el Centro de Escritura de la Universidad del Cauca (CEUC), de Colombia, como el Centro de Escritura de la Universidad de Tarapacá (CEDEUTA), de Chile, explicitan en su objetivo la intención de potenciar y fortalecer las habilidades de lectura junto con las de escritura (Chois, 2015; Uribe y Gascón, 2015). Por otro lado, el Centro de Escritura Académica y Pensamiento Crítico CEAPC, de la Universidad de Las Américas Puebla, en México, considera que uno de sus públicos objetivos son aquellos estudiantes de primera lengua que presenten problemas de lectoescritura (Corte Velasco, 2015); es decir, sí supone la importancia del desarrollo de la habilidad de comprensión lectora, pese a ser desde una perspectiva remedial. No obstante, ninguno de esos tres lugares incluye esta labor dentro de la descripción de los servicios prestados a los estudiantes, ni explica las dinámicas desde las que se lo abarca, de ser así. Otro caso es el Centro de Escritura 
Javeriano de Bogotá, que incluye una preocupación por la relación de la lectura en la educación superior y media como parte integral del concepto de su centro de escritura, pese a que únicamente desde su eje de investigación; es decir, se omite en sus otros dos ejes: formación y servicios (González, 2015).

Adicionalmente, llama la atención que, de los quince centros y programas presentados en el libro de Molina, solo tres hayan tenido en cuenta, para su denominación, algún término derivado del ejercicio de la lectura. Sin embargo, así como en los casos descritos anteriormente, ninguno de estos hace referencia expresa al trabajo de esta habilidad en la descripción de los servicios prestados para los estudiantes (Murillo, 2015; Casillas, 2015; Sánchez y Hugo, 2015). Respecto a lo anterior, téngase también en cuenta que estos quince centros y programas presentados en el libro de Molina constituyen la mitad de los miembros activos de la Red Latinoamericana de Centros y Programas de Escritura (RLCPE, s. f.), y, de ellos, además de los tres mencionados, solo otros seis incluyen términos relacionados con la lectura a la hora de nombrar estos espacios.

Así las cosas, particularmente en Latinoamérica esta desatención a la competencia lectora por parte de los centros y programas de escritura resulta paradójica en tanto durante la última década se ha evidenciado un notorio y creciente interés por las prácticas de lectura -aunadas a las de escritura- desde las instituciones de educación superior de la región (Natale, 2013), lo que ha traído consigo un aumento de iniciativas de enseñanza, investigación, publicación, eventos y esfuerzos de sistematización (Navarro y otros, 2016). Es decir que la región ha presentado una historia y un desarrollo importante de las prácticas de comprensión lectora, respaldado por evidencias como la existencia de la revista Lectura y vida, publicación en la que una respetable cantidad de especialistas aportaron al tema durante la década de los ochenta, una de las razones por las cuales durante este tiempo Latinoamérica se caracterizó "por un interés temprano en la lectura como objeto de investigación y enseñanza» (Navarro, 2016, 44).

Desde esta región, tal menor atención a la lectura en los centros y programas podría atribuirse al hecho de que estos lugares son aún un fenómeno reciente, en crecimiento y evolución. Prueba de ello es que, aunque el primer centro de escritura (de la Universidad Interamericana de Puerto Rico) del que se tiene evidencia en Latinoamérica data de algunos años antes de comenzar el siglo XxI, el segundo (del ITAm de México) se consolidó solo hasta el año 2005 (Molina, 2014); es decir, hace tan solo un poco más de una década. Por tanto, resulta más que probable que aún haya mucho terreno por recorrer. Aunado a ello, y a propósito de la experiencia norteamericana respecto de los programas y centros de escritura, resulta probable, así mismo, que esta desatención de Latinoamérica se deba a una influencia de la literatura y experiencias estadounidenses, que no colocan en el centro la lectura, como otras iniciativas más autóctonas. Para respaldar esto, Navarro (2016) explica que, a diferencia de la tradición estadounidense, y gracias al desarrollo y la influencia de las Ciencias del Lenguaje en Latinoamérica, el concepto de discurso académico juega un papel fundamental en la región; mientras que en la tradición 
norteamericana de estudios de la composición y la retórica predomina el concepto de "escritura». Esto, explica el autor, constituye una diferenciación clara respecto a cómo se investiga y enseña: "[...] mientras que el concepto de "discurso" construye un objeto de investigación en tanto producto textual estructurado a partir de un sistema lingüístico subyacente, el concepto de "escritura" propone un objeto de investigación en tanto proceso situado que involucra dimensiones sociales, históricas, psicológicas, educativas y también lingüísticas» (Navarro, 2016, 44).

Pero, sean cuales sean las razones, lo claro es que las evidencias mencionadas muestran que, en efecto, ya existe un interés -o al menos atención- por apoyar la habilidad de comprensión lectora desde estos espacios de apoyo a la escritura, aunque estas prácticas aún no se han hecho expresas y poco se han difundido como ejemplo de aplicación. Y tal falta de socialización en el marco específico de estos espacios lleva a señalar que, hasta ahora, poco se ha podido "aprender» de las prácticas al respecto. Todo ello apunta a que, si bien se percibe una cierta atención a la comprensión lectora desde unos pocos centros o programas de escritura, esta aún se asume como otro servicio, mas no como un elemento inseparable e indispensable del ejercicio escrito académico.

\section{DE LO LINGÜíSTICO A LO FUNCIONAL}

Para intentar explicar las raíces de este vacío, cabe recaer en lo obvio, y es que la escritura ha sido siempre el apoyo que los asistentes más demandan al acudir a estos lugares, además porque, como se mencionó arriba, estos se originaron en Norteamérica (Molina, 2014); es decir, a partir de sus intereses y necesidades. Además, habrá que recordar que sus inicios o proliferación estuvieron motivados por sucesos como crisis de alfabetización de una primera lengua, como en Norteamérica con las admisiones abiertas (Carino, 1996; Calle Arango, Pico y Murillo, 2017; Boquet, 1999); en Latinoamérica, por los vacíos que se consideraba eran dejados en la preparación de la Educación Media (Carlino, 2002; Moyano, 2004; Pérez Abril y Rincón Bonilla, 2013), las altas tasas de deserción y «el proceso de masificación del ingreso a la educación superior y de expansión de los sistemas universitarios" (Navarro, 2016, 38) durante las últimas décadas; o bien necesidad de refuerzo de una segunda lengua, como sucedió en Asia (Tzu-Shan, 2017; Hays, 2010). De ahí, como se sabe, se le adjudicó originalmente un valor remedial a estos lugares, que recaía en vacíos de índole lingüística, para los cuales no se requería un desarrollo de pensamiento crítico o establecimiento de fines comunicativos.

Por lo mismo, hay una difusión de la idea de la relevancia del producto escrito -que la escritura como proceso ha intentado desvirtuar, pero que continúa siendo ampliamente difundida en el imaginario académico-, razón por la cual uno de los problemas más comunes que enfrentan estos espacios es la idea de que se solicita ayuda para ser corregido. Pensamiento que ni en Asia ni en Europa (Tan, 2011; Johnston, Cornwell y Yoshida, 2010; Tzu-Shan, 2017; Voigt y Girgensohn, 2015), 
ni en Estados Unidos (North, 1984; Howard y Schendel, 2009), ni en Latinoamérica (Molina, 2014; Calle Arango, Pico y Murillo, 2017) ha logrado desaparecer.

Quizás por el arraigo de estas dos razones tampoco se ha evidenciado la necesidad de trabajar la comprensión lectora, como sí ha pasado con la escritura: bajo la idea remedial, los mismos estudiantes son quienes, esperando tener mejores resultados, acuden para obtener un apoyo que llene de forma inmediata sus vacíos específicos. Pero estas dificultades o necesidades pocas veces se vinculan con los procesos de la comprensión lectora, cuando en realidad son sus raíces (Griswold, 2006; Bañales y otros, 2015); esta desvinculación resulta un pensamiento difundido no solo entre los estudiantes, sino también entre los profesores, que poco ha cambiado desde la queja presentada por Stephen North (1984) a finales del siglo pasado. En esa misma línea, esta desatención hacia la comprensión lectora desde la idea de tales lugares puede relacionarse con lo que Tan (2001) considera el resultado de una inadecuada atención por parte del docente, nacida a partir de una visión reduccionista que concibe la escritura como una habilidad independiente de aquellas relacionadas con la lectura, la conversación y la escucha. Adicionalmente, esta realidad puede comprenderse desde la vieja idea de que la escritura era un ejercicio intelectual que debía ser ejercido a partir de unas normas específicas, de manera que su valoración recaía en el adecuado manejo de la sintaxis, la ortografía, etc. Se trataba de un ejercicio mecánico.

Este punto de vista limitado a lo meramente lingüístico se quedó corto a lo que es el uso del lenguaje y la escritura como herramienta social y de comunicación, de manera que, como se sabe, esta visión es posteriormente ampliada con la noción de competencia comunicativa de Hymes. Con esta última, tal como señala Zanón (1990), el dominio de la lengua se dejó de concebir como un conocimiento del sistema formal subyacente al lenguaje, y se empezó a asumir que era mediante el conocimiento lingüístico que se adquiría la capacidad para vincular el conocimiento formal de la lengua con los diferentes contextos de comunicación. En términos simples, hacer uso del lenguaje de manera adecuada en las diferentes situaciones y escenarios que el hablante enfrenta, tanto en su vida laboral como personal.

Si bien entonces la competencia comunicativa se mide a partir de lo que se necesita saber para poder desenvolverse de manera adecuada dentro de una comunidad hablante (Richards y Rodgers, 1999), lejos de hablarse de un conocimiento más, se trata de la concepción de una herramienta social a ser desarrollada con objetivos que desbordan lo académico y que, por tanto, generan la necesidad de conocimientos que desbordan lo meramente gramatical. Y estos cambios, sin duda, no pasaron desapercibidos por los centros y programas de escritura. Aunque bautizar estos espacios como laboratorios, clínicas o centros de escritura presuponía, en principio, que estos habían de enfatizar en el ejercicio mismo de la producción escrita, cuando comienza a haber una noción de la competencia comunicativa, en el que la escritura no debe únicamente ser correcta sino funcional en términos pragmáticos, queda al descubierto la imposible desvinculación entre la escritura y otros ejercicios de pensamiento crítico y reflexivo, así como su relación con la formación profesional: 
En esta sociedad en la que prima el valor de la capacidad intelectual, conocimiento, desarrollo científico y tecnológico y de la capacidad de innovación como factores básicos de competitividad y de supervivencia, como elementos claves para el desarrollo económico, social y el mejoramiento de las condiciones de vida, bienestar individual y colectivos la educación de las personas se convierte en un asunto estratégico, siendo fundamental el desarrollo de ciertas competencias como la capacidad de pensar, la independencia intelectual y el aprendizaje autónomo (Pardo, 2015, 77).

Este ejercicio intelectual y de pensamiento necesariamente pasa por ese conducto que es el lenguaje, a partir del cual toman forma las visiones, las perspectivas y los conocimientos, que luego deberán ser comunicados al mundo, de nuevo mediante el uso del lenguaje mismo. Particularmente en Latinoamérica, fue hacia el 2001 que se "planteó por primera vez la idea de que la lectura y la escritura debían ser objeto de enseñanza en la universidad, no como un asunto remedial sino como la responsabilidad de las instituciones educativas de compartir las prácticas lectoras y escritoras propias de cada ámbito disciplinar»(Carlino, 2013, 358).

Con ello, el papel de los centros y programas de escritura deja de limitarse a uno remedial de llenar vacíos lingüísticos; su papel en la formación académica se complejiza, pues debe velar por que todos los estudiantes desarrollen su competencia comunicativa para desenvolverse adecuadamente dentro de una comunidad hablante. Esto implica pensar ya no en el ejercicio de escritura, sino en un ejercicio de pensamiento crítico, con sus complejidades: «... si se niega su carácter intrínseco comunicativo no se estaría enseñando la lengua, sino solamente el sistema de la lengua, que es inerte» (Flotts, Manzi, Polloni, Carrasco y Zambra, 2016, 12). Poder comunicarnos es lo que nos convierte en seres sociales, capaces de hacer parte de comunidades hablantes, de manera que el lenguaje es también una herramienta misma de supervivencia.

\section{LECTURA LÚDICA: ÚTIL PERO INSUFICIENTE}

Algunos de estos espacios han tenido la iniciativa de ofrecer la modalidad de clubes de lectura (Núñez, 2013) y otros escenarios que ofrecen la posibilidad de involucrarse con este ejercicio desde una perspectiva lúdica. Posiblemente tales iniciativas tienen fundamento en esa valoración -cuyo auge se dio a mediados del siglo $\mathrm{xx}$ - de la individualidad dentro de los procesos educativos, del estudiante como sujeto activo; y, por tanto, de la importancia de sus factores motivacionales. Lo anterior basado en que un alumno con motivación aprende con mayor facilidad; y, de hecho, muchas de las dificultades de aprendizaje se deben justamente a bloqueos afectivos (Piaget, 2005), por lo que parte del éxito en su aprendizaje se desprende de cómo este perciba tales procesos.

Una manera de fomentar esto es justamente motivar el ejercicio de la lectura desde los gustos individuales de los asistentes al centro o programa de escritura, y alejar de lo académico los objetivos de tal ejercicio. La lectura por placer tiene 
un efecto significativo tanto en el desarrollo de habilidades de lectura como en la adquisición del lenguaje (Griswold, 2006). Ello resulta fácil de ofrecer desde los centros y programas de escritura, pues son un espacio en el que se le puede otorgar al estudiante la posibilidad de explorar libremente sobre las estrategias, mediante un ejercicio de lectura que se adecúe a los intereses particulares, de manera que le encuentren sentido. Es decir, estos espacios pueden no presentar una limitación de tipo temático -como sucede en las asignaturas, que deben ceñirse a aquellas relacionadas con temas específicos-. Esta posibilidad de los centros y programas de escritura de moverse libremente entre diferentes temáticas y géneros, a diferencia del desarrollo de una clase, responde al hecho de que el desarrollo de la habilidad lectora puede ser asumido como el objetivo en sí mismo, y no como el medio para acceder a algo más.

Tales dinámicas resultan convenientes para el entorno mismo de los centros y programas de escritura, en donde los estudiantes pueden hablar con libertad y sin miedo a equivocarse -lo que los motiva a compartir libremente- pues no hay nota o medida que valore sus intervenciones. Esta resulta una idea interesante también en la medida en que este tipo de diálogos entre pares potencia el pensamiento y ayuda a desarrollar la capacidad de comunicación desde la espontaneidad, recordando además los evidentes beneficios que el trabajo colaborativo ofrece en los procesos de aprendizaje (Casillas, Cabezas y Hernández, 2017). Al respecto, Colomer explica que la discusión en grupos enriquece la comprensión en tanto que ofrece interpretaciones variadas, refuerza la memoria a largo plazo y ayuda a mejorar la comprensión profunda, así como el pensamiento crítico (Colomer, 1997). Entonces, se podría pensar que, si hay un propósito y una real motivación para hacerlo, que involucra la posterior puesta en común de las ideas, estos procesos se lograrán de manera natural, pues, tal como señala Solé (1996), la actividad intelectual que se activa al momento en que se intenta comprender un texto -la necesidad de priorizar, seleccionar, valorar, integrar con previos conocimientos, así como construir nuevos esquemas- conlleva que mediante el ejercicio lector se dé un aprendizaje, aunque este no haya sido el objetivo original de la lectura.

Aunque estas iniciativas resultan interesantes y útiles, no son suficientes. De asumir la competencia lectora únicamente desde la noción de habilidad transferible, genérica y desvinculada de las necesidades y especificidades disciplinares, estos espacios corren el riesgo de omitir parte de su responsabilidad con la formación académica. En otras palabras, si bien los centros y programas de escritura pueden tomarse libertades al margen del currículo, también lo es que tienen una responsabilidad con la institución y la alfabetización académica. Lo que se busca decir, entonces, no es que se deba negar la importancia de iniciativas como los clubes de lectura u otras semejantes, sino que, así mismo, resulta imperativo abordar esta habilidad desde la didáctica en educación superior, con miras a un apoyo a los procesos alfabetizantes en la academia. 


\section{UNA RESPONSABILIDAD CON EL CURRÍCULO}

Hacia los ochenta y noventa, y desde las percepciones de la crisis educativa, en Latinoamérica se dio una "multiplicación de iniciativas de enseñanza de la escritura académica en un contexto de masificación del acceso a la educación superior» (Navarro, 2016, 40). Estas buscaban, en su mayoría, solventar las dificultades de escritura en los estudiantes y resultan insuficientes para los paradigmas actuales. Por un lado, porque el desarrollo de la competencia comunicativa debe dirigirse no solo a los más atrasados sino a todos los estudiantes (Calle Arango, Pico y Murillo, 2017), y tales talleres de escritura masivos están, por lo general, "orientados a la nivelación de carencias formativas previas y a la enseñanza de habilidades básicas de escritura académica, y mayormente a cargo de profesores que provenían de las ciencias del lenguaje, en general con poca formación específica en didáctica de la escritura académica..." (Navarro, 2016, 41). Por otro lado -aunque por la misma razón-, estos cursos propedéuticos muchas veces no permean el currículo, pues el foco recae sobre habilidades genéricas más que en la alfabetización en géneros académicos y propios de las esferas profesionales involucradas. No en vano, respecto de estos cursos intensivos en Norteamérica, pues allá también se generó este fenómeno, Bazerman y otros señalan que «el enfoque del curso intensivo de escritura puede criticarse por segregar la escritura dentro de cursos seleccionados específicamente en lugar de integrarla en todos los cursos" (Bazerman y otros, 2016, 188).

Por lo anterior resulta clave recordar que el término alfabetización académica, que para Latinoamérica fue importado por Paula Carlino comenzando el siglo XxI, se relaciona íntimamente con el aprendizaje situado y la responsabilidad de las instituciones, desde todas las asignaturas que componen el currículo, de favorecer la participación de los estudiantes en las culturas escritas propias de la esfera profesional en la que pretendan formarse (Carlino, 2013). En este sentido, "Introducir de manera sistemática y progresiva a los estudiantes en la lectura y producción de géneros académicos -orales y escritos- así como de los que deberán producir en su vida profesional aparece como uno de los objetivos centrales en los estudios superiores...» (Natale y Moyano, 2006, 2). Por ello se considera determinante el involucramiento de las demás disciplinas en esta labor, pues familiarizarse con los contenidos de cada materia consiste tanto en apropiarse de su sistema conceptualmetodológico como de sus prácticas discursivas características (Rosales y Vásquez, 2011; Carlino, 2002), lo que requiere un esfuerzo institucional integral.

Por lo mismo, y más aún en las últimas décadas, como apoyo a las habilidades transversales de comunicación, y como parte integral de la formación académica, los centros y programas de escritura han buscado apoyar a todas las áreas que componen el currículo. Evidencia de ello es la íntima relación que se ha establecido, desde los noventa, con el movimiento de la Escritura a través del Currículo (WAC). Tal movimiento, originado en la década de los setenta en Norteamérica, busca que los docentes de las diferentes asignaturas que componen el currículo «involucren a sus estudiantes de un modo más profundo en la labor disciplinar 
que necesariamente se hace mediante la escritura» (Bazerman, 2016, 34). En pocas palabras, este movimiento se puede definir como una "... apuesta teórica y práctica por la transformación o complejización de esos cursos generales de escritura a configuraciones disciplinarmente informadas, colaborativas entre expertos en escritura y expertos en contenidos disciplinares, curricularmente híbridas y diversas, y con presencia en diferentes tramos de la formación obligatoria de las carreras» (Navarro, 2016, 40). Con ello se pone en evidencia la importancia de la implementación de estrategias de desarrollo de habilidades comunicativas en las asignaturas que componen el currículo, y no al margen de estas. Y para tal tarea, ningún aliado más adecuado que los centros y programas de escritura (Molina, 2012), pues tanto estos lugares como este coinciden en su tendencia «... a rechazar el enfoque de enseñanza de escritura de tipo general o transversal para todo contexto y, en su lugar, se esfuerzan por identificar diferencias disciplinares en escritura..." (Bazerman y otros, 2016, 189).

\section{LA LECTURA AL SERVICIO DE LA ALFABETIZACIÓN}

Ya señalaban Bazerman y otros que «La práctica en Escribir a través del Currículum y la investigación en Escribir en las Disciplinas han tenido que enfrentar el hecho de que la mayor parte de la escritura académica está fuertemente vinculada a la lectura y hace referencia permanente a esta» $(2016,62)$. En este orden de ideas, resultaría obtuso concebir todavía el ejercicio de la escritura como un elemento aislado de las demás habilidades relacionadas con el lenguaje, y pretender estar aportando significativamente a una formación transversal en la educación superior a través de un apoyo únicamente en esta área. Este, por el contrario, debe asumirse como parte integral de algo mayor: el lenguaje como herramienta de comunicación y desempeño social, lo que implica el desarrollo de otras capacidades cognitivas. Risemberg (1996, en Bazerman y otros, 2016) halló evidencias de una relación directa entre la calidad de los textos que producen los estudiantes con el tiempo que se tomaron para leer la información relacionada con el trabajo. Por tanto, si en la actualidad los centros y programas de escritura buscan permear las asignaturas que componen el currículo, deben pretender formar lectores críticos como paso previo al desarrollo de buenos escritores.

Pero, si bien en el marco de la alfabetización académica el papel de la comprensión lectora al servicio de la escritura es evidentemente fundamental, también debe insistirse en el rol de la lectura en sí misma dentro de la preparación profesional del estudiante en tanto a la formación de un individuo con pensamiento crítico. Lo anterior dado que el desarrollo de un pensamiento crítico que conduzca al individuo a una competencia comunicativa adecuada implica pensar en la puesta en práctica de procesos previos y posteriores a la mera codificación y decodificación de signos escritos. Y justamente la práctica sistemática de una adecuada lectura conlleva desarrollar tanto el lenguaje como, más importante aún, el pensamiento, en la medida que implica subprocesos que involucran capacidades tales como la reflexión, la 
consciencia y el espíritu crítico (Rodríguez Vives y Puebla Soler, 2016; Narvaja de Arnoux, Di Stefano y Pereira, 2002). En este orden de ideas es que la UNESCO señala que la lectura se concibe como un instrumento, una manera de pensar (Flotts, Manzi, Polloni, Carrasco y Zambra, 2016), y por ende como un medio para construirnos a nosotros mismos como personas invidivuales, con experiencias y perspectivas propias.

Esto queda reforzado por la concepción más reciente de la comprensión lectora, que parte de una visión constructivista, a partir de la cual se hace evidente que leer, más que meramente comprender un mensaje, resulta de una interpretación en la que tienen alta incidencia los conocimientos previos: "Partimos de la idea de que leer es un acto interpretativo que consiste en saber guiar una serie de razonamientos hacia la construcción de una interpretación del mensaje escrito a partir tanto de la información que proporciona el texto como de los conocimientos del lector» (Colomer, 1997). A razón de ello, las consecuencias que se desprenden de leer un texto trascienden significativamente un ejercicio aislado de comprensión: "La lectura, como hemos visto, constituye tanto un fin como un medio que resulta fundamental para ampliar las posibilidades y oportunidades de desarrollo individual y de la sociedad en general» (Márquez, 2015, 13). Es por esto que se insiste en que el ejercicio de lectura se constituye como parte integral de una visión de mundo, en tanto que involucra previos saberes, tales como conocimientos, opiniones, ideas o creencias (Rodríguez Vives y Puebla Soler, 2016; Ballester y Ibarra, 2015). Por lo mismo, el ejercicio, una y otra vez, desemboca en una experiencia y un proceso completamente individual, que parte de particularidades y converge en resultados irrepetibles e intransferibles entre individuos.

Muy en consonancia con ello, Daniel Cassany explica la importancia de hacer del estudiante una persona crítica, "... que mantiene una actitud beligerante en la consecución de sus propósitos personales, a través de la lectura y la escritura, pero también la que participa de modo constructivo en el desarrollo de una comunidad plural, respetuosa y progresista» (Cassany, 2003, 114). Se trata, entonces, de formar individuos capaces de participar e intervenir activamente en una comunidad hablante, defender sus posturas desde un pensamiento crítico, hacerse con el conocimiento; es decir, servir a sus intereses como ser individual, y a los de la comunidad como ser social, teniendo en cuenta que "gracias a la interacción social las personas aprenden los significados y símbolos, poniendo en juego la capacidad distintivamente humana de pensamiento» (Puc y Ojeda, 2018, 9). En este sentido, la lectura se constituye en un medio a partir del cual podemos acceder a los conocimientos y participar activamente en los entornos sociales, puesto que el mundo letrado en el que vivimos tiende a complejizarse cada vez más (Flotts, Manzi, Polloni, Carrasco y Zambra, 2016). Se trata, pues, de una herramienta, no de un conocimiento aislado del que se podría prescindir.

No en vano la educación superior se ha considerado el momento en que la alfabetización académica toma un matiz sociopolítico importante: con su mayoría de edad, el individuo debe asumir responsabilidades civiles en las que su 
comunicación y manejo del lenguaje tiene repercusiones en campos como el legal y democrático (Calle Arango, 2018). El uso del lenguaje otorga al individuo la posibilidad de desenvolverse como ser social; pero implica, así mismo, la responsabilidad que conlleva hacer parte de esta sociedad, y su enseñanza debe corresponder a ello: "...los estudiantes que inician una carrera universitaria se enfrentan al aprendizaje de las prácticas letradas nuevas, las propias de la disciplina que empiezan a estudiar, sea ingeniería, derecho, periodismo o cualquier otra. Estas prácticas son complejas, utilizan discursos altamente especializados y exigen saber construir significados de modo específico, de acuerdo con una tradición preestablecida...» (Morales y Cassany, 2018, 71). Como consecuencia, el apoyo desde las prácticas de lectoescritura en el marco de la alfabetización académica no puede ni debe limitarse a actividades lúdicas, remediales o genéricas. Así mismo, cabe tener en cuenta que las características y fundamentos propios de estos centros y programas de escritura ofrecen ciertas ventajas sobre las prácticas tradicionales que pueden -y deben- ser aprovechadas para fomentar otros elementos clave dentro de la formación del estudiante, como la autonomía y el establecimiento de alianzas con los docentes de las diferentes asignaturas.

\section{LA AUTONOMÍA DESDE LA COMPRENSIÓN LECTORA}

La autonomía es un concepto que ha tomado significativa fuerza desde los setenta y ochenta del siglo pasado. Knowles (1975) fue uno de los primeros que teorizó al respecto y es a él a quien se le debe la distinción entre aprendizaje dirigido por el profesor (teacher directed learning) y el aprendizaje autodirigido (selfdirected learning), que desembocará en el conocido término aprender a aprender (capacidad de autodirección). Con ello, quedó claro que el individuo debía tener la capacidad de guiar sus propios procesos de aprendizaje; y, por tanto, ser responsable de ellos (Holec, 1989). Respondiendo al panorama actual, en el cual las tecnologías han asumido un papel preponderante dentro de los procesos educativos, la idea del estudiante autónomo no deja más que tomar fuerza en los diferentes países (Torrano, Fuentes y Soria, 2017), pues se evidencia la necesidad de implementar estrategias que ofrezcan conocimientos sobre cómo aprender, más que sobre lo que hay que aprender. Esto se relaciona con la idea de que "Lograr que los alumnos sean responsables de su aprendizaje nos lleva a la concepción del alumno durante toda la vida" (De la Antonia, 2013, 71). Por ello, resulta atinado que haya centros y programas -como el de la Universidad de los Andes de Colombia- que ya ofrezcan material pedagógico, como lo son guías de lectura, mediante su plataforma web, como recursos que el estudiante puede utilizar para mejorar sus habilidades. Esto teniendo presente que no puede limitarse únicamente a este tipo de difusión, sino que debe haber otros medios de apoyo que atiendan las necesidades particulares de los estudiantes y los ayuden a descubrir cuáles son las mejores estrategias según las especificidades de los géneros académicos y profesionales. Ello recordando que esta capacidad de aprender a aprender es una habilidad que requiere de guía 
práctica; no es intuitivo o innato (Torrano, Fuentes y Soria, 2017), sino que debe desarrollarse de manera guiada.

Así, está comprobado que, mediante un adecuado desarrollo de las estrategias de comprensión de lectura crítica, se puede potenciar la autonomía del estudiante (Solé, 1996), pues, a través del establecimiento de objetivos y la posterior valoración de estos, se generará una consciencia sobre los propios procesos cognitivos. Igualmente, un estudiante que dimensione las ventajas de acceder a la lectura como parte de un proceso de obtención de conocimiento y acceso al mundo -el general y el de la información- convergerá en un mejor desenvolvimiento sus labores académicas y en su desempeño personal: «... el estudiante debe tomar conciencia de cómo es su aprendizaje, se refiere al proceso que ocurre en el individuo para el reconocimiento, aceptación y apropiación del conocimiento» (Pardo, 2015, 72).

En este sentido, el fomento de una práctica lectora crítica resulta imprescindible dentro de las labores de centros y programas de escritura, a los cuales los estudiantes acuden no para ser corregidos, sino para obtener herramientas que a futuro les sirvan para, de manera independiente y autónoma, utilizar en las situaciones comunicativas a las que se enfrenten. La ventaja clave que se presenta es ser un lugar en el que se puede seguir su propio ritmo y establecer sus propios objetivos, dado su carácter de espacio permanentemente abierto y en acción. Será el estudiante quien decida durante cuánto tiempo desea trabajar, sobre qué, de qué manera, en un lugar donde se estará en constante disposición para apoyarlo individualmente en su desarrollo de estrategias que fomenten su autonomía a la vez que se motiva la generación de pensamiento crítico en un proceso autodirigido.

\section{SINERGIAS ENTRE DOCENTES}

Mediante la posibilidad de trabajo individual y mayor desarrollo de autonomía que estos espacios pueden ofrecer al implementar prácticas de lectura, no estará replicando modelos de otras clases, ni haciendo el trabajo de otros docentes; al contrario, el establecimiento de alianzas con estos otros docentes debe hacer parte del apoyo prestado. Esto, en primer lugar, porque, como señala Carlino (2003), una de las mayores dificultades para los estudiantes cuando se enfrentan a los textos académicos y científicos es que estos están dirigidos a lectores especializados y no a personas en proceso de formación, razón por la cual se requiere, para lograr un verdadero desarrollo de la lectura crítica, una disposición de los demás docentes para "compartir con los alumnos la cultura académica que los profesores han adquirido como miembros de sus comunidades disciplinares» (Carlino, 2003, 5). Lo anterior en tanto que la aproximación de los estudiantes a los textos académicos y científicos es nueva, y «... cuyo discurso es necesariamente objeto de aprendizaje para quienes se proponen ingresar a la universidad y proveen un marco para el uso del aprendizaje en ese contexto»(Moyano, 2004, 115). 
Adicionalmente, esta familiarización incluye no solamente un proceso de inmersión para conocer estos códigos, sino también el desarrollo de diferentes estrategias de lectura que respondan a propósitos y objetivos -académicos y/o profesionales- diversos: "Aprender a leer y escribir géneros discursivos exige dominar cómo se dicen las cosas, pero también saber qué se tiene, a quién, con qué propósito, cómo se argumenta y cómo se organiza el discurso" (Morales y Cassany, 2018, 72). Estas formas específicas de lectura, a su vez, varían según las áreas temáticas (Bazerman y otros 2016), de manera que, por ejemplo, se puede ser competente a nivel lector en una disciplina y no serlo en otra. Y si bien es a partir del establecimiento de los objetivos de cada tarea planteada en las asignaturas que variarán las formas de lectura (Bazerman y otros, 2016, 62), es en la preparación y planteamiento de estas tareas donde se debe dar un trabajo conjunto entre los docentes y los colaboradores de los centros y programas de escritura, sobre todo porque es común que a los estudiantes se les exija -sin enseñárseles- la capacidad de comprensión de textos propios de culturas escritas y campos de estudio que son nuevos para ellos (Carlino, 2003); es decir, obviando el proceso de enculturación en el que se les debería sumergir al entrar a la educación superior.

Esta sinergia entre las asignaturas y los centros o programas de escritura resulta clave en tanto que, tal como señala Moyano, asignar esta responsabilidad a un equipo formado por el experto en lengua y el docente de la disciplina conduce a una suerte de dinámica de negociación entre pares en donde ambos aportan sus conocimientos y se complementan para el proceso de enseñanza (Moyano, 2010). Con ello se solventará el inconveniente que se presenta frecuentemente respecto del hecho de que «... los propios profesores no somos conscientes de que analizar un texto consiste en implementar este conjunto de operaciones cognitivas y por ello pedimos a los alumnos analizar lo que leen pero sin darles precisiones sobre cómo hacerlo» (Carlino, 2003, 20). Sinergias como estas en términos de escritura ya existen; tal es el caso de los cursos asociados del Centro DIGA en el CESA (Colombia), en los que se ofrece «asesoría para la planeación, desarrollo y calificación de diferentes actividades que involucren habilidades lingüísticas» (Murillo, 2012), el centro de escritura de la Universidad de los Andes y PRODEAC (Moyano, 2010), pero se requiere de su multiplicación en asuntos que se refieren a la lectura. Es decir, que se amplíe y se haga expreso aquello que, como se mencionó al principio, el Centro de Escritura de la Universidad Javeriana de Cali profesa en cuanto a ofrecer asistencia a los profesores en la puesta en marcha de estrategias de lectura en sus asignaturas (Núñez, 2013).

\section{CONClusiones}

Pese a la copiosa literatura sobre las prácticas en los centros y programas de escritura, poco se ha enfatizado en la necesidad o ejercicio de la comprensión lectora como parte integral del proceso de desarrollo de la competencia comunicativa. Sin embargo, también es verdad que este interés ha aumentado en los últimos años, 
lo que se evidencia en la mención que de ello hacen algunos de estos espacios en diferentes lugares geográficos. Al margen de las razones por las cuales haya sucedido esto, dados los procesos que involucra el ejercicio de la comprensión lectora en el desarrollo de la competencia comunicativa y la capacidad crítica del individuo, se hace un llamado urgente a que se asuma esta habilidad como parte clave de los servicios presentados por estos espacios. Lo anterior en tanto que, si bien estos espacios buscan aportar a la alfabetización académica desde una perspectiva comunicativa, habrá que tener en cuenta que parte indiscutible de esta es, justamente, la comprensión lectora: «La literacidad reconoce el proceso de la lectoescritura como una práctica social, lo que implica además de trabajar la lectura y escritura como procesos lingüísticos y psicológicos, como prácticas socioculturales» (Flotts, Manzi, Polloni, Carrasco y Zambra, 2016, 13). No en vano Núñez, tras su breve contextualización sobre los inicios y el auge de los centros de escritura, define el objetivo de estos espacios así: "Ayudar a los estudiantes universitarios de cualquier curso con aspectos relacionados con la escritura y la lectura" (Núñez, 2013, 67).

Así las cosas, con miras a formar un estudiante crítico y apoyar de manera transversal las diferentes asignaturas que componen los programas de educación superior, actualmente los centros y programas de escritura tienen la responsabilidad no solo de que los estudiantes escriban bien, sino de que desarrollen sus habilidades comunicativas y pragmáticas. Para esto se requiere que los centros y programas de escritura, en consonancia con los cursos propedéuticos y otros esfuerzos que se están haciendo dentro del currículo en el marco de la educación superior en diferentes instituciones a nivel internacional (como el ofrecimiento de cursos específicos sobre escritura académica, talleres nivelatorios a recién ingresados, entre otros), adopten mayores prácticas que apoyen la comprensión lectora de los estudiantes en formación profesional, y con ellas los procesos cognitivos que a partir de una lectura sistemática se pueden alcanzar.

Adicionalmente, por las condiciones mismas de los centros y programas de escritura, estas prácticas tienen la ventaja de poder ser abarcadas desde metodologías que fortalezcan la autonomía de los estudiantes, pues en estos espacios se desdibujan las limitaciones temáticas, temporales y académicas que muchas veces no favorecen una formación individualizada, al ritmo de cada estudiante. Así mismo, resulta más que pertinente que los colaboradores de estos espacios establezcan alianzas con los docentes de las demás asignaturas que componen el currículo, de manera que se pueda permear directamente en el uso y prácticas específicas de las comunidades hablantes que están en juego.

Para cerrar, resulta igualmente imperativo que se dé una socialización de las experiencias que los centros y programas de escritura tengan al respecto, de manera que se pongan a consideración las diferentes perspectivas y resultados, con miras a solventar, en equipo, un vacío que hoy en día están presentando estos espacios. 


\section{REFERENCIAS BIBLIOGRÁFICAS}

BALLESTER, J. e IBARRA, N. (2015) La formación lectora y literaria en contextos multiculturales. Una perspectiva educativa e inclusiva. Teoría de la Educación. Revista Interuniversitaria, 27 (2), 161-183.

Bañales, G.; Vega, N.; Araújo, N.; Reyna, A. y Rodríguez, B. (2015) La enseñanza de la argumentación escrita en la universidad: Una experiencia de intervención con estudiantes de Lingüística aplicada. RMIE, 20 (66), 879-910.

Bazerman, C. (2016) Prólogo a la edición en español, en Bazerman, C.; Little, J.; Bethel, L.; Chavkin, T.; Fouquette, D. y Garufis, J. Escribir a través del Currículum. Una guía de referencia. Córdoba, Universidad Nacional de Córdoba, 32-37.

Bazerman, C.; Little, J.; Bethel, L.; Chavkin, T.; Fouquette, D. y Garufis, J. (2016) Escribir a través del Currículum. Una guía de referencia. Córdoba, Universidad Nacional de Córdoba.

Boquet, E. (1999) «Our Little Secret»: A History of Writing Centers, Pre-to Post-Open Admissions. College Composition and Communication, 50 (3), 463-482.

Bruffee, K. (2008) Peer Tutoring and the "Conversation of Mankind", en BARNeTt, R. y Blumer, J. The Longman Guide to Writing Center Theory and Practice. Great Britain, Pearson, 206-218.

Calle Arango, L. (2018) Educación superior: la alfabetización en géneros discursivos. Educação \& Realidade, 43 (2), 629-651.

Calle Arango, L.; Pico, A. L. y Murillo, J. H. (2017) Los centros de escritura entre nivelación académica y construcción de conocimiento. Cadernos de pesquisa, 47 (165), 872-895.

CARINO, P. (1996) Open Admissions and the Construction of Writing Center History: A Tale of Three Models. The Writing Center Journal, 17 (1), 30-48.

CARLINO, P. (2002) ¿Quién debe ocuparse de enseñar a leer y a escribir en la universidad?: tutorías, simulacros de examen y síntesis de clase en las humanidades. Lectura y Vida, 23 (1), 6-14.

CARlino, P. (2003) Leer textos científicos y académicos en la educación superior: obstáculos y bienvenidas a una cultura nueva. Uni-Pluri/Versidad, 3 (2), 17-24.

Carlino, P. (2013) Alfabetización académica diez años después. Revista Mexicana de Investigación Educativa, 18 (57), 355-381.

Casillas, S. (2015) Centro de lectura y redacción, en Molina, V. (ed.) Panorama de los centros y programas de escritura en Latinoamérica. Cali, Pontificia Universidad Javeriana de Cali, 87-92.

Casillas, S.; CABEZAS, M. y Hernández, A. (2017) Construcción de conocimiento colaborativo mediado tecnológicamente: aportaciones teóricas desde el análisis de prácticas educativas. Teor. Educ., 29 (2), 61-86.

Cassany, D. (2003) Aproximaciones a la lectura crítica: teoría, ejemplos y reflexiones. Tarbiya, 32, 113-132.

Chols, P. (2015) Centro de Escritura Universidad del Cauca Ceuc, en Molina, V. (ed.) Panorama de los centros y programas de escritura en Latinoamérica. Cali, Pontificia Universidad Javeriana de Cali, 39-43.

Colomer, T. (1997) La enseñanza y el aprendizaje de la comprensión lectora. Signos. Teoría y Práctica de la Educación, 20, 6-15.

Corte Velasco, C. (2015) Centro de Escritura Académica y Pensamiento Crítico CEAPC, en Molina, V. (ed.) Panorama de los centros y programas de escritura en Latinoamérica. Cali, Pontificia Universidad Javeriana de Cali, 55-61. 
De la Antonia, A. y Souto, M. (2013) Estrategias para fomentar la autonomía del estudiante, en VJornadas de Formación para Profesores de Español en Chipre. Chipre, 68-92. Consultado el 19 de marzo de 2018. goo.gl/2h9sVj.

Flotts, P.; Manzi, J.; Polloni, P.; Carrasco, M. y Zambra, C. (2016) Aportes para la enseñanza de la lectura. Santiago de Chile, unESCO.

GonzÁlez, B. Y. (2015) Centro de Escritura Javeriano Puj Bogotá, en Molina, V. (ed.) Panorama de los centros y programas de escritura en Latinoamérica. Cali, Pontificia Universidad Javeriana de Cali, 63-70.

Griswold, G. (2006) Postsecondary Reading: What Writing Center Tutors Need to know. Journal of College Reading and Learning, 37 (1), 61-72.

HARRIS, M. (1988) SLATE (Support for the Learning and Teaching of English) Statement: The Concept of A Writing Center. The National Council of Teachers of English. Consultado el 19 de marzo de 2018. goo.gl/kcSAAN.

HAYs, G. (2010) Learners helping learners in an EFL writing center. JALT2009 Conference Proceedings, 589-596.

Holec, H. (1989) Nouveaux roles des enseignants, en ANDrÉ, B. (selec.) Autonomie et enseignement/apprentissage des langues étrangéres. Paris, Didier-Hatier, 147-150.

Howard, S. y SCHENDEL, E. (2009) Making the Writing Center a Writing Environment. Student Summer Scholar, Paper 30.

Johnston, S.; Connwell, S. y Yoshida, H. (2008) Writing Centers in Japan. 日本における Writing Centers, 181-192.

Johnston, S.; Cornwell, S. y Yoshida, H. (2010) Writing centers and tutoring in tutoring in Japan and Asia. JALT2009 Conference Proceedings, 692-701.

KNowles, M. (1975) Self-Directed Learning. Cambridge, Cambridge Adult Education.

Márquez, A. (2015) Editorial. Sobre lectura, hábito lector y sistema educativo. Perfiles Educativos, 39 (155), 3-18.

Molina, V. (2012) Escritura a través del currículo en Colombia: situación actual y desafíos. Magis, 5 (10), 93-118.

Molina, V. (2014) Centros de escritura: una mirada retrospectiva para entender el presente y futuro de estos programas en el contexto latinoamericano. Legenda, 18 (8), 9-33.

Molina, V. (ed.) (2015) Panorama de los centros y programas de escritura en Latinoamérica. Cali, Pontificia Universidad Javeriana de Cali.

Moore, R. H. (1995) The Writing Clinic and the Writing Laboratory, en MurPHY, C. y Law, J. (eds.) Landmark Essays on writing centers. California, Hermagoras, 3-10.

Morales, O. A. y CASSANY, D. (2018) Leer y escribir en la universidad: Hacia la lectura y la escritura crítica de géneros científicos. Revista Memoralia, (5), 69-82.

Moyano, E. I. (2004) La escritura académica: una tarea interdisciplinaria a lo largo de la currícula universitaria. Revista Texturas, 4 (4), 109-120.

MoyANO, E. I. (2010) Escritura académica a lo largo de la carrera: Un programa institucional. Revista Signos, 43 (74), 465-488.

MuriLLo, J. (2012) La universidad y los procesos de lecto-escritura: centro de apoyo para la lectura, la oralidad y la escritura DIGA. Panorama, 6 (10), 87-97.

Murillo, J. H. (2015) Centro de apoyo para la Lectura, la Oralidad y la Escritura DigA, en Molina, V. (ed.) Panorama de los centros y programas de escritura en Latinoamérica. Cali, Pontificia Universidad Javeriana de Cali, 25-32.

Narvaja de Arnoux, E.; Di Stefano, M. y Pereira, C. (2002) Representaciones sociales de la lectura, en NARvaja de ARnoux, E.; Di Stefano, M. y Pereira, C. La lectura y la escritura en la universidad. Buenos Aires, Universidad de Buenos Aires. 
NATALE, L. (2013) Integración de enfoques en un programa institucional para el desarrollo de la escritura académica y profesional. Revista Mexicana de Investigación Educativa, 18 (58), 685-707.

Natale, L. y Moyano, E. (2006) Evolución de las conceptualizaciones sobre el lenguaje escrito como herramienta para el aprendizaje en algunos profesores de materias universitarias, en RODI, A. M. y CASCO, M. (coords.) Casco, Lengua-Investigación. Actas Primer Congreso Nacional "Leer, Escribir y Hablar Hoy". Buenos Aires, Universidad Nacional del Centro de la provincia de Buenos Aires. Consultado el 16 de marzo de 2018. goo.gl/nsFNSB.

Navarro, F. (2016) Introducción, en Bazerman, C.; Little, J.; Bethel, L.; Chavkin, T.; FouQUETTE, D. y GARUFIS, J. Escribir a través del Currículum. Una guía de referencia. Córdoba, Universidad Nacional de Córdoba, 38-45.

Navarro, F.; Ávila, N.; TAPia, M.; Cristovão, V.; Moritz, M. E.; NArváez, E. y Bazerman, C. (2016) Panorama histórico y contrastivo de los estudios sobre lectura y escritura en educación superior publicados en América Latina. Revista Signos. Estudios de Linguística, 49 (1), 100-126.

NorTH, S. (1984) The idea of a writing center. College English, 46 (5), 433-446.

NúÑEZ, J. A. (2013) Una aproximación a los centros de escritura en Iberoamérica. Legenda, 17 (17), 63-102.

PARDO, R. T. (2015) Empoderamiento de la evaluación en el aprendizaje autónomo. Revista Ciencias de la Educación, 26 (46), 71-82.

Pérez Abril, M. y Rincón Bonilla, G. (coord.) (2013) ¿Para qué se lee y se escribe en la universidad colombiana? Un aporte a la consolidación de la cultura académica del país. Bogotá, Pontificia Universidad Javeriana.

PiAget, J. (2005) Inteligencia y afectividad. Buenos Aires, Aique.

Puc, L. y OJEDA, M. (2018) Promoviendo la lectura en ámbitos extraescolares: un círculo para adolescentes en Coscomatepec, Veracruz, México. Álabe, 17, 1-20. Consultado el 23 de marzo de 2018. goo.gl/S7drZk.

Richards, J. C. y RoDgers, T. S. (1999) Approaches and Methods, en Language Teaching. A description and analysis. Cambridge, Cambridge University Press (15 edition).

RLCPEC (s. f.) Integrantes. Consultado el 16 de marzo de 2018. goo.gl/LepiHT.

Rodríguez Vives, M. y Puebla Soler, M. (2016) La comprensión de lectura en la enseñanza del español como lengua extranjera. Pedagogía Universitaria, 21 (2), 81-93.

Rosales, P. y VÁzquez, A. (2011) Leer para escribir y escribir para aprender en la educación superior. El caso de la escritura académico argumentativa a partir de fuentes. Contextos de Educación, 11 (11), 1-13.

SÁnchez, V. y Hugo, E. (2015) Programa de Lectura y Escritura Académica PleA, en Molina, V. (ed.) Panorama de los centros y programas de escritura en Latinoamérica. Cali, Pontificia Universidad Javeriana de Cali, 117-126.

Solé, I. (1996) Estrategias de comprensión de la lectura. Curso de especialización en Lectura $y$ Escritura. Buenos Aires, Lectura y Vida/Asociación Internacional de Lectura.

TAN, B. H. (2011) Innovating writing centers and online writing labs outside North America. Asian EFL Journal, 13 (2), 390-417.

Torrano, F.; Fuentes, J. L. y SORIA, M. (2017) Aprendizaje autorregulado: estado de la cuestión y retos psicopedagógicos. Perfiles Educativos, 39 (156), 160-173.

TZu-SHAn, C. (2017) The idea of a writing center in Asian countries: A preliminary search of models in Taiwan. Praxis: a writing center Journal. 
Uribe, P. y Gascón, R. (2015) Centro de Escritura Universidad de Tarapacá Cedeuta, en Molina, V. (ed.) Panorama de los centros y programas de escritura en Latinoamérica. Cali, Pontificia Universidad Javeriana de Cali, 45-53.

Voigt, A. y Girgensohn, K. (2015) Peer Tutoring in Academic Writing with Non-Native Writers in a German Writing Center-Results of an Empirical Study. Journal of Academic Writing, 5 (1), 65-73.

ZANÓN, J. (1990) Los enfoques por tareas para la enseñanza de las lenguas extranjeras. Cable, $5,19-27$. 